Journal of Animal

Ecology 2003

72, 725-735

\title{
Variable performance of individuals: the role of population density and endogenously formed landscape heterogeneity
}

\author{
CATHERINE A. PFISTER* and SCOTT D. PEACOR $\uparrow$ \\ *Department of Ecology and Evolution, 1101 E. 57th St., University of Chicago, Chicago, IL 60637, USA; and $\dagger$ Great \\ Lakes Environmental Research Laboratory (NOAA), Ann Arbor, MI and Department of Fisheries and Wildlife, \\ Michigan State University, East Lansing, MI, USA
}

\begin{abstract}
Summary
1. Individuals can show positive correlations in performance (e.g. growth and reproduction) through time beyond the effects of size or age. This 'performance autocorrelation' has been attributed previously to traits that differ among individuals or to extrinsic generators of environmental heterogeneity.

2. A model of mobile consumers on a dynamic resource showed that consumer foraging gave rise to resource heterogeneity that in turn generated autocorrelation in growth in consumers.

3. Resource heterogeneity and growth autocorrelation were most pronounced when consumers were poorer foragers, moving locally and with an imperfect ability to identify the highest resource cells.

4. The model predicted that lowered population density enhanced resource heterogeneity and the strength of growth autocorrelation.

5. Consistent with model predictions, an experiment with tidepool limpets demonstrated that autocorrelation in growth changed with population density, with individuals in lower density tidepools showing stronger temporal correlations in growth.

6. Our model and empirical results contrast with those of previous studies with plants, where dominance and suppression increases with increasing density.

7. Our results suggest that growth autocorrelation can occur without invoking sizedependent advantages, intrinsic trait differences or extrinsic generators of environmental heterogeneity.
\end{abstract}

Key-words: consumer-resource interactions, growth autocorrelation, individual variation, individual-based model, limpets, patchiness, Tectura scutum.

Journal of Animal Ecology (2003) 72, 725-735

\section{Introduction}

Variation in the performance of individuals in natural systems is ubiquitous (Darwin 1859; Lomnicki 1988). Although much of this variation can be explained by age and size (e.g. Deevey 1947; Caswell 2001; Coulsen et al. 2001), many individuals can show positive correlations in performance through time beyond the effects of age or size (Hatch 1990; Fraser et al. 2001; Pfister \& Stevens 2002). For example, differences in the quality of breeding sites or breeding experience may cause individual differences in the breeding success of Northern Fulmars (Hatch 1990). Furthermore, the familiar

Correspondence: C. A. Pfister, Department of Ecology and Evolution, 1101 E. 57th Street, University of Chicago, Chicago, IL 60637, USA. Tel. 773834 0071; Fax: 773702 9740; E-mail: cpfister@uchicago.edu pattern of 'dominant' and 'suppressed' individuals in populations of terrestrial plants (Schmitt, Ehrhardt \& Cheo 1986, 1987; Weiner et al. 2001) suggests that some individuals have persistently superior performance compared with others.

While variability in individual performance independent of size is fundamental to evolutionary theory, it has received far less attention in ecological studies. However, ecological theory suggests that variation in individual performance can affect the dynamics of populations and how we model them. Variation in individual growth or reproduction can affect the extinction risk of populations (Lomnicki 1980; Connor \& White 1999; Uchmanski 2000; Fox \& Kendall 2002; Kendall \& Fox 2002; Pfister \& Stevens 2003), particularly when some individuals have persistently better performance. For example, the persistent superior performance of some individuals may ameliorate extinction risk (e.g. 
726

C. A. Pfister \&

S. D. Peacor

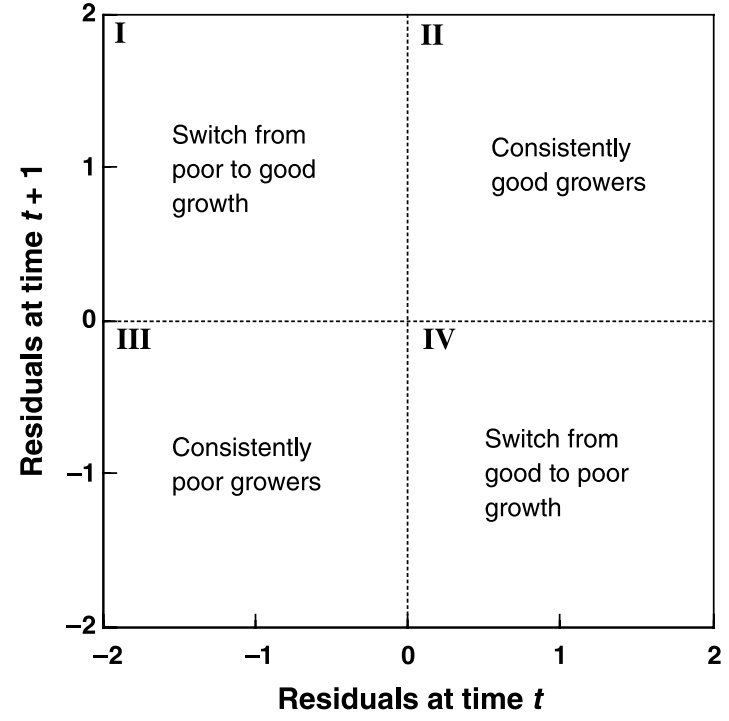

Fig. 1. The method used to estimate growth autocorrelation illustrated graphically. The residuals from a relationship between size at time $t+1$ and growth at time $t+1$ are plotted as a function of the residuals from a relationship between size at time $t$ and growth at time $t$. A significant positive correlation indicates that individuals have consistently poor or good growth and primarily occupy quadrants II and III.

Connor \& White 1999; Kendall \& Fox 2002). Further, quantifying variation in individual performance is an important factor in determining whether individualbased models are better descriptors of population dynamics (DeAngelis et al. 1993; Pfister \& Stevens 2003). Recent theory suggests that growth autocorrelation can lead to great deviations from classical model predictions of size structure, including highly skewed size distributions (Pfister \& Stevens 2002). For all the above reasons, it is essential to quantify and understand the origin of variation in individual performance.

One method to quantify individual performance independent of size is to analyse whether performance, independent of size, is consistently different among individuals over finite time steps. Pfister \& Stevens (2002) quantified the correlation in growth rate over time using the residuals from a regression of growth on size. This estimate, termed 'growth autocorrelation', demonstrated a positive correlation in growth rates through time in a kelp (Alaria nana) and a predatory gastropod (Nucella canaliculata). Briefly, to quantify and test for growth autocorrelation (Fig. 1), the correlation in residuals from multiple size vs. growth regressions through time is estimated. Thus, if there are two or more intervals over which growth is estimated, the residuals at each sequential time interval are tested for a significant positive relationship.

Growth autocorrelation has been suggested to arise through two broad mechanisms (Pfister \& Stevens 2002). First, the differences may be intrinsic to the organism due to traits that differ among individuals and have either a genetic (e.g. Arnold 1981) or learned
Journal of Animal

Ecology, 72,

$725-735$ (e.g. Palmer 1983, 1984; West 1986; Werner 1992;
Dukas \& Bernays 2000) basis. In contrast, extrinsic generators of environmental heterogeneity might drive differences among individuals if, for example, prey resources were differentially available among individuals or factors that determined resource productivity, such as water or nutrients, varied in the landscape. Extrinsic factors may be especially important for sessile species or those with reduced mobility where resource heterogeneity can be persistent through time.

Here we propose, based on the results of an individualbased simulation model, that a third mechanism generates growth autocorrelation. The foraging activities of consumers can generate resource heterogeneities in the landscape. This causes differential foraging opportunities to develop and growth autocorrelation among consumers can then arise. What is qualitatively different about this mechanism compared with previously discussed mechanisms is that growth autocorrelation arises without intrinsic differences in individual traits controlling foraging activities and in an initially homogeneous environment.

Understanding the origin of performance correlations can uncover how organisms interact with their resources and the abundance and distribution of conspecifics. Although there has been a great deal of attention focused on how variation in individual size changes as a function of population density (e.g. Wilbur \& Collins 1973; Harper 1977; Lomnicki 1988; Peckarsky \& Cowan 1991; Ziemba \& Collins 1999), little attention has been paid to how density alters individual variability in growth and the existence and magnitude of performance correlations. For example, it is unknown whether there are strictly size-dependent processes driving increased variability among individuals, or whether intrinsic trait differences are expressed to a greater extent at higher densities. If strictly sizedependent processes such as differential survivorship or growth dominate, then growth autocorrelation would be estimated to be near zero. In contrast, growth autocorrelation is predicted to be stronger at higher density if traits that affect foraging activity are expressed more differentially at higher density. An example might include differential abilities to garner resources at low resource levels. The observation of dominant and suppressed individuals in plant studies suggests that growth autocorrelation does increase with density (Harper 1977; Cousens \& Hutchings 1983; Weiner 1985; Schmitt et al. 1986, 1987; Dean, Thies \& Lagos 1989; Reed 1990; Weiner et al. 2001). Studies with animals also suggest that growth autocorrelation may increase at higher densities, based on increased variability in size among individuals (Wilbur \& Collins 1973; Rubenstein 1981, Peckarsky \& Cowen 1991). Although none of these studies quantified growth autocorrelation, the existence of highly variable and skewed size distributions may suggest the presence of growth autocorrelation based on models and demographic data from field populations (Pfister \& Stevens 2002). Ultimately, understanding the origin of growth 
Resource heterogeneity and individual variability
(C) 2003 British

Ecological Society, Journal of Animal Ecology, 72, 725-735 autocorrelation will allow us to determine how density affects individual performance differences and their manifestation at the population level.

In this study we examine growth autocorrelation and the effect of population density on its magnitude, both theoretically with an individual-based model, and empirically through the manipulation of limpet density in intertidal pools.

\section{Methods}

\section{FIELD DENSITY MANIPULATION EXPERIMENT}

We quantified individual differences in growth in the limpet Tectura scutum (Rathke) (= Notoacmea scutum) in the context of a field density manipulation. The experiment was carried out at two rocky headlands on the Washington state shoreline east of Neah Bay, Washington $\left(48^{\circ} 23^{\prime} \mathrm{N}, 124^{\circ} 35^{\prime} \mathrm{W}\right)$, where T. scutum is the dominant limpet in high intertidal pools. T. scutum is not territorial and does not occupy home scars. Individuals can and do move within tidepools, although much of their movement appears to be during high tide when the pools were inundated. Ten pairs of tidepools were chosen based on similarity in size and tidal height. All pools ranged between 1.2 and $1.5 \mathrm{~m}$ above MLLW, a chart datum for the multi-year mean of the lower low water heights for the area (Gill \& Schultz 2000). From each pair of tidepools, one was chosen randomly to be a control, while $T$. scutum densities were reduced by half in the other. The few individuals of other limpet species that were encountered in all pools (Lottia pelta (Rathke), L. digitalis (Rathke) and L. Strigatella (Rathke)) were removed. The density reduction treatment was reduced to $n=9$ because sand deposition filled one pool, killing most of the limpets. Limpets were removed in the reduced density treatment without changing the variability in size structure. Limpet length was measured at the widest dimension of the shell base. All T. scutum greater than $9 \mathrm{~mm}$ were tagged individually with numbered bee tags (Chr. Graze, Germany) placed posteriorly on the shell with epoxy (Z-Spar ${ }^{\mathrm{TM}}$, Kop-Coat, Rockaway, NJ, USA) in May. In total, 878 individuals were marked in the 19 experimental tidepools. The manipulations were performed over three spring and summer intervals during $1998(n=3$ pairs of pools) $1999(n=4)$ and $2000(n=3)$. T. scutum was remarkably site-specific and there were only a few instances where individuals migrated to another tidepool.

Limpets were censused and remeasured at low tide at least four times until September each year; growth was assayed as a descriptor of performance and was estimated from changes in length. To control for size distribution differences among tidepools, a linear regression of size vs. growth (the best fit) was performed with all individuals and the residuals were used to express size-independent estimates of growth. The approximately 4-month duration of the experiment was divided into two intervals in which growth was estimated. The experiment was not divided into finer time intervals to minimize measurement errors. The growth was analysed pairwise among tidepools, with a linear regression of size vs. growth for each pair of pools. A mean estimate for residuals per tidepool thus provides an indication of growth after size effects are removed. Maintaining the paired structure of the tidepools and analysing residuals also controlled for any year effects. Growth autocorrelation was then estimated as the slope from a regression of the residuals of growth from the second interval on the first (Pfister \& Stevens 2002). Growth autocorrelation therefore defines the degree to which differences in individual limpet growth are correlated over time, independent of the effects of size.

\section{CONSUMER-RESOURCE MODEL}

We explored whether consumer foraging patterns could provide a general explanation of growth autocorrelation with an individual-based model of dynamic consumer-resource interactions. We asked specifically how different foraging patterns, comprised of both the ability to identify high resources and the ability to move, and consumer density affected the strength of growth autocorrelation. We used the model to link resource patterns with consumer growth and growth autocorrelation. We chose an individual-based simulation approach to the problem because it was essential that we be able to manipulate both the foraging patterns of individuals and identify the performance of individuals, as well as examine the consequences of local interactions between consumers and their resource.

We manipulated the foraging patterns of individuals with parameters that controlled the probability that individuals moved and their ability to find high resource cells. We modelled a grid $(75 \times 75$ square cells $)$ of dynamic resources that grew logistically following the equation:

$$
R_{i_{t+1}}=r R_{i_{t}}\left(1-\frac{(r-1) R_{i_{t}}}{K}\right)
$$

where $R_{i_{t}}$ is the amount of resource at time $t$ in cell $i$. Although initially we used a range of values for the rate of resource growth $r$ and the carrying capacity $K$, we report model runs where $r$ was set at 1.004 and $K$ was set at $10^{7}$ for each cell. These parameter values prevented total resource depletion while allowing enough resources for consumers to grow in size.

We allowed a fixed number of individual consumers to forage on this grid in order to track individual consumer trajectories through time. The probability that consumers moved $(m)$ or remained in the same cell was manipulated. Movement was restricted to one of nine cells, eight neighbouring cells and the currently occupied cell. We also varied the probability that an individual that moved selected a randomly chosen cell or 
728

C. A. Pfister \&

S. D. Peacor the cell with the highest resource level $(g)$. In the latter case, if multiple cells shared the highest resource level, then a cell among these was chosen at random. Thus, the frequency of movement and the ability to find high resource cells was manipulated independently. The grid was designed as a torus so that all cells had eight surrounding cells. Individual consumer growth during a time step $(t)$ was described by the equation: Growth $=$ $a M_{t}^{b}$, where $a$ is a product of the conversion efficiency of food to growth $(c)$, a consumption coefficient ( $e$ ) and the level of resources in the cell $i\left(R_{i}\right)$. Although $R_{i}$ defined the total amount of resources per $i$ cell, $20 \%$ of $K\left(2 \cdot 0 \times 10^{6}\right)$ always remained as a refuge, preventing local resource extirpation and mimicking the ability of resources to regenerate in the presence of consumers (e.g. Kitting 1980). The consumption coefficient defined the fraction of available resources $\left(R_{i}-2 \cdot 0 \times 10^{6}\right)$ eaten by an individual. The conversion rate and the consumption coefficient were constant $\left(3 \cdot 0 \times 10^{-9}\right.$ and $0 \cdot 1$, respectively) and equal for all individuals to eliminate any size-independent trait variation among individuals. A negative value of $b(-0 \cdot 5)$ was used to scale growth to mass $(M)$ to approximate the negative sizedependent growth seen in limpets in the experiment. Thus, the amount of resources an individual consumed was a function of its mass and the resources available. Although the simulation was designed such that no individual could consume more than $80 \%$ of the carrying capacity $(K)$, consumption in our scenarios varied only from $6 \cdot 0$ to $11 \cdot 3 \%$ of the available resources at the end of the simulations. Individuals were drawn initially from a normal distribution with a mean mass of $1 \cdot 0$ and a variance of $0 \cdot 1$. Resources in each cell averaged $3 \cdot 0 \times 10^{6}$ at the beginning of each model run. At each time step, each consumer grew in relation to its size and the resources available and consumed on its cell. After consumption, consumers then moved in accordance with the parameters $m$ and $g$. Although $m$ and $g$ usually covaried, we also tested the effect of frequent movement (high $m$ ) without the ability to identify high resource patches (low $g$ ) to explore further the link between foraging patterns and growth autocorrelation. When more than one consumer moved to a cell in a time step, the individual that 'ate' first was determined randomly. The model was written using the SWARM modelling platform (http:// www.swarm.org).

The model was run until there were no transitory dynamics and the resource patterns remained relatively constant (2000 time steps). Resource heterogeneity was quantified as a spatial covariance at one, two, four and eight cells distant to describe the spatial pattern of resource disparity (Rossi et al. 1992). Although spatial correlation can also be used to describe spatial relationships, we chose not to use them here because correlation, unlike covariance, is normalized to the overall variance (Rossi et al. 1992). Thus, covariance describes better the absolute range of resource values, and it is the absolute value of the resources $\left(R_{i}\right)$ that drives growth rates in the consumers. Our covariance estimates thus provide a metric of the disparity among cells in the model landscape.

The growth processes of a random subset of 100 consumers ( 72 in the lowest density scenario) was analysed from each model run over finite time intervals, starting at $t=2000$. For each set of parameter combinations, we quantified how much variation in growth among individuals was explained by size $(M)$ vs. previous growth. A linear model described best the relationship between size and growth. Residuals were saved from a linear regression of growth on size for three sequential time steps beginning at $t=2000$. Growth autocorrelation was quantified by regressing the residuals from the second interval on the residuals from the first interval, analogous to the methods described for the field experiment. The number of individuals on the resource grid varied over a factor of 8 , from 72 to 576 , to explore how density affected resource distribution and the processes that contribute to variation in growth.

To understand better the link between the spatial variation in resource levels and autocorrelation in growth, the resources encountered by consumers were analysed in several ways. First, the variability in the mean resource level encountered in the eight cells surrounding a subset of 80 consumers was quantified for each model run. Neighbouring resources levels were also averaged over 10 and 50 time-periods to probe the persistence of resource differences encountered by individuals through time. Secondly, individual consumer growth was analysed as a function of local resources and its movement pattern (e.g. $m$ and $g$ in the model) to elucidate how resource heterogeneity and movement contributed to variation in growth.

\section{Results}

\section{FIELD DENSITY MANIPULATION EXPERIMENT}

Limpets at reduced densities grew at more than twice the rate of limpets at control densities (mean $=1.05 \mathrm{~mm}$ per month $\pm 0.24 \mathrm{SE}$ and $0.51 \pm 0 \cdot 09$, respectively, $t=2 \cdot 846, P=0 \cdot 019)$, while growth variability did not differ in the two treatments (CV in growth $=124 \cdot 0 \pm$ $34 \cdot 7$ for the reduced density treatment vs. $148 \cdot 3 \pm 20 \cdot 9$ for controls, $t=1 \cdot 127, P=0 \cdot 289)$. Residual values for growth also were significantly smaller on average in control pools (Fig. 2a, $t=3 \cdot 578, P=0 \cdot 007$ ). One criticism of statistical tests with residuals is that residuals may be intercorrelated and heteroscedastic (GarćiaBerthou 2001). As an alternative analysis, ANCOVAs for each pool pair were also estimated using density as a factor and limpet length as the covariate. Overall, limpet growth was always negatively related to shell length, regardless of treatment. In all 10 ANCOVAs the slopes were not heterogeneous and in eight of 10 pool pairs the limpets in the reduced density treatment grew significantly greater than the controls. Thus, the slope
Journal of Animal

Ecology, 72,

$725-735$ 
729

Resource

heterogeneity and individual

variability
(C) 2003 British

Ecological Society, Journal of Animal Ecology, 72, $725-735$
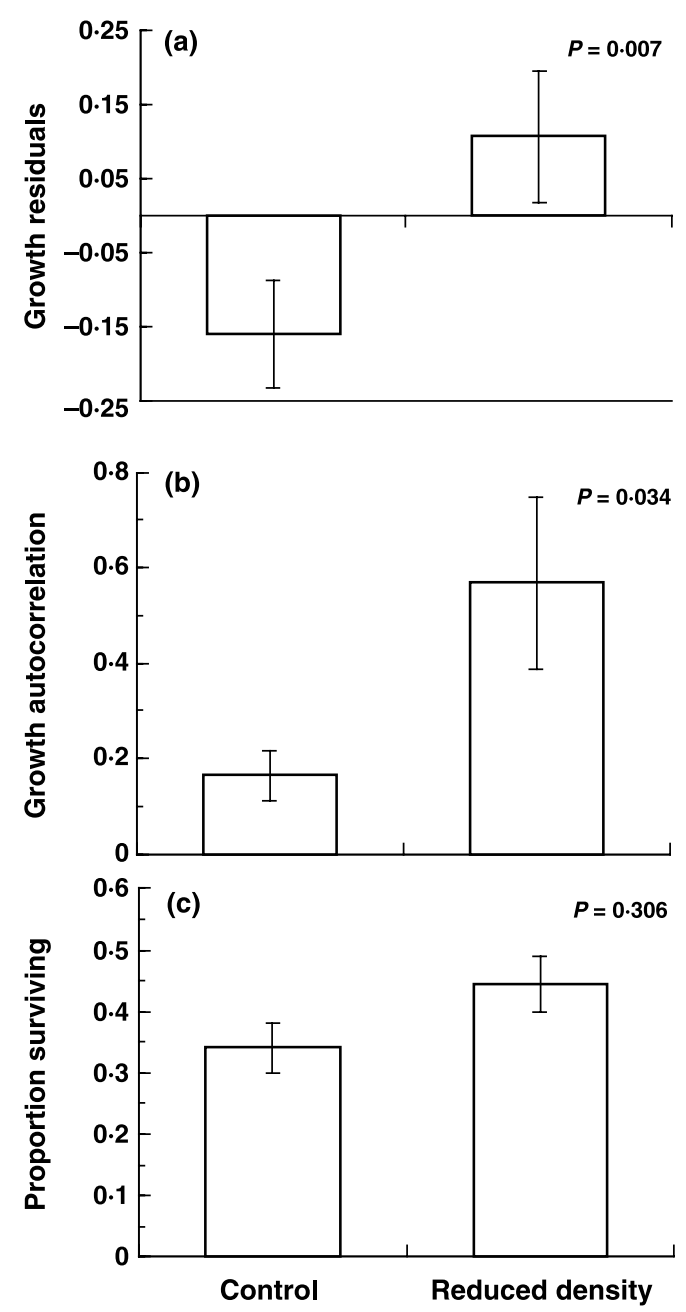

Fig. 2. The results of an experimental density manipulation with the limpet $T$. scutum showing the effects of reduced density on (a) mean growth, (b) growth autocorrelation, and (c) survivorship. Mean growth estimates are the residuals from a regression of length vs. growth. Growth autocorrelation describes the temporal relationship among residuals. Standard errors are shown for 10 control tidepools and nine where density was reduced; the $P$-values are based on paired $t$-tests.

of the relationship between size and growth was unchanged by the treatment, indicating that growth at reduced density was higher for limpets of all sizes, a result analogous with the residual analysis in Fig. 2a.

Density had an effect on growth autocorrelation opposite to that inferred from previous empirical work. Individual limpets at reduced densities showed a significantly greater positive autocorrelation in growth through time (mean growth autocorrelation across tidepools $=0.568)$ compared with individuals in the higher density control tidepools $(0 \cdot 164$, Fig. 2 b, $t=2 \cdot 545, P=0.034)$. Survivorship over 4 months did not differ significantly between treatments (Fig. 2c, $t=1 \cdot 094, P=0 \cdot 306$ ), indicating that density differences were maintained throughout the experiment. Thus, there was strong autocorrelation in growth among individuals, independent of size and these correlations were dependent on density, increasing in strength more than threefold when density was reduced. Overall variability in growth did not differ between the treatments; rather, how the variation was expressed among individuals is what differed. That is, limpets that grew more relative to others in a pool did so consistently through time, and this pattern was more pronounced in pools with lower density.

\section{CONSUMER-RESOURCE MODEL}

The model showed that both the effectiveness and the density of the forager changed spatial heterogeneity and 'patchiness' in the resource and this affected the strength of growth autocorrelation. When foragers were restricted in movement, 'patches' of four to eight cells in length developed (Fig. 3a-c). Here, we define 'patches' to be regions of high or low resource levels that developed during simulations, as described by higher covariance estimates. Although these patches were dynamic structures across the landscape, the overall landscape structure (i.e. the patchiness) was persistent. When the consumer population was composed of poor foragers (i.e. those that moved and found the best neighbouring resource cells with a low probability $(m=0 \cdot 2, g=0 \cdot 2))$ resource levels among cells were most variable and patchiness was pronounced (Fig. 3). As the probability increased that individuals moved and found high resource cells, the difference in resource levels among cells decreased and patchiness declined (contrast Fig. 3a with Fig. 3b,c).

The development of resource patchiness on the landscape was enhanced by both the inability of consumers to find the best local cell and by restricted movement. As evidence for this, when consumers were allowed to move over the entire grid but had a low probability of finding cells with the highest resource density $(m=1 \cdot 0$, $g=0 \cdot 2$ ), patches did not develop as observed with individuals that foraged locally. Rather, resources were more homogeneous and there was no correlation in resource magnitude in neighbouring cells, as indicated by relatively low resource covariance measures across all cell distances (see 'highly mobile' foragers, Fig. 3d). Using this same set of parameters for foraging but specifying movement to only neighbouring cells restores the heterogeneity in the landscape, with resource results nearly identical to those for the 'intermediate forager' in Fig. 3.

Our concurrent analysis of individual consumer growth showed distinct patterns of variability. As foraging ability declined, the $\mathrm{CV}$ of individual growth rate increased (Fig. 4a). Growth autocorrelation was especially important to explaining this variation in growth as consumer foraging ability declined (Fig. 4b). In contrast, as consumers became better foragers and resources heterogeneity declined (Fig. 3), growth was less variable and size alone was the best predictor of growth (Fig. 4a,b). Consumers with the ability to move anywhere on the landscape at each time but with a relatively low probability of finding the best resources 
730

C. A. Pfister \&

S. D. Peacor (a)

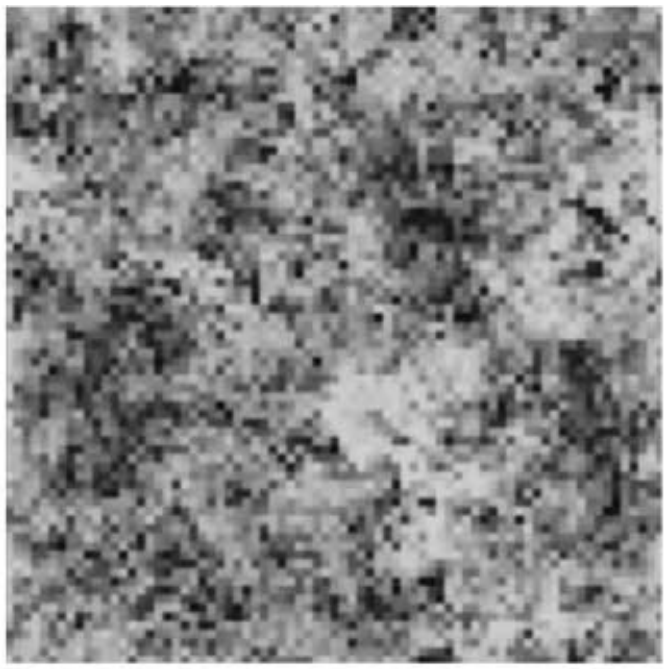

(c)

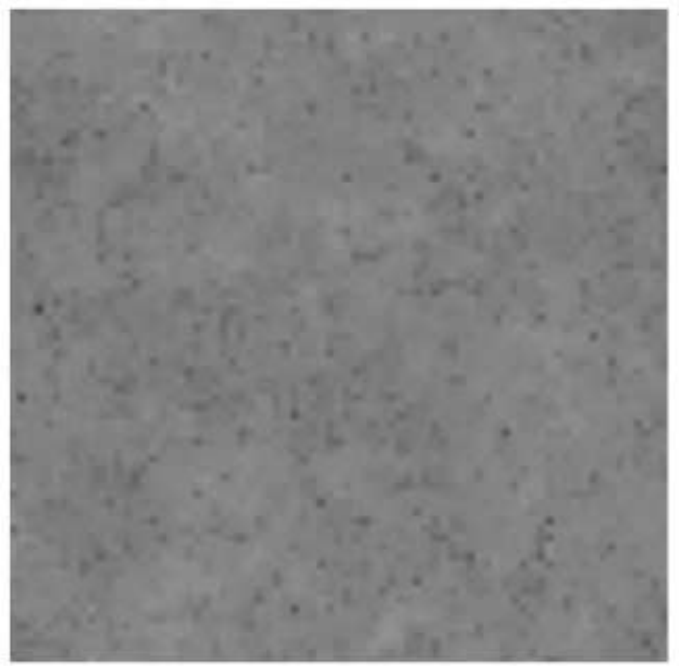

(b)

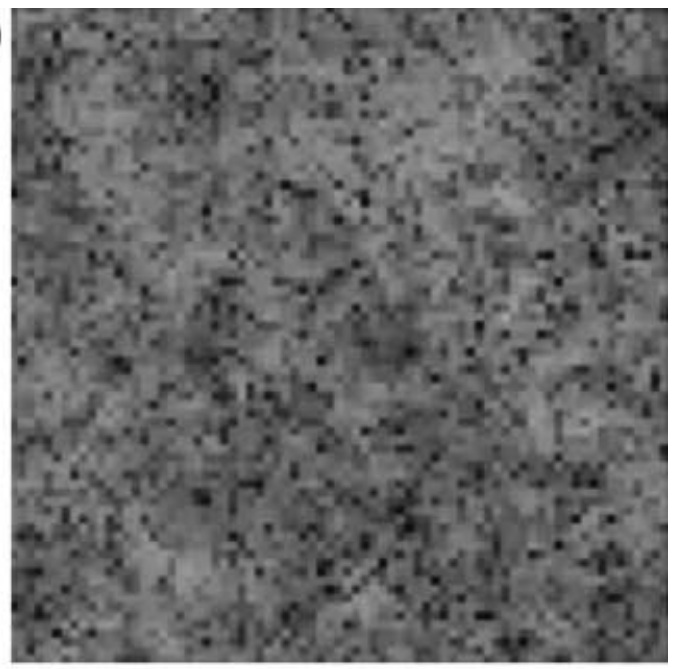

(d)

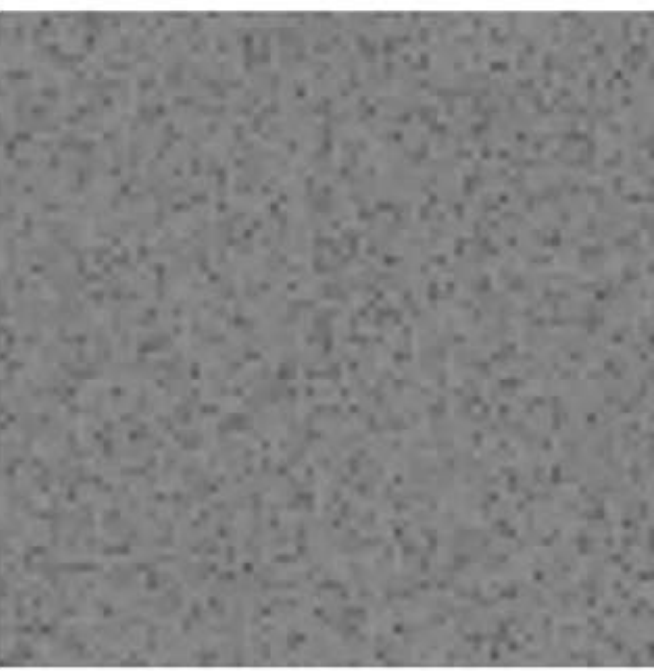

(e)

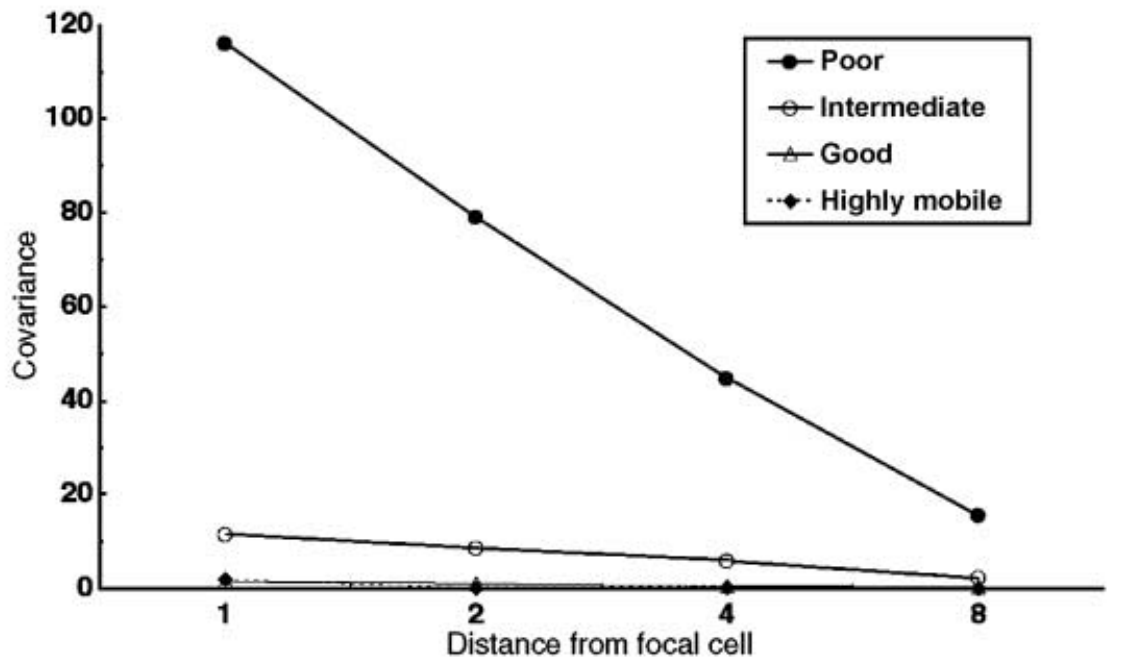

Fig. 3. Model output when individuals were (a) poor foragers (probability of moving $(m)$ and finding the best cell $(g)=0 \cdot 2)$ and

(C) 2003 British

Ecological Society, Journal of Animal Ecology, 72,

$725-735$ (b) intermediate foragers ( $m=0 \cdot 5, g=0 \cdot 5)$, (c) good foragers $(m=0 \cdot 8, g=0 \cdot 8)$ and (d) 'highly mobile' foragers, or those that can move anywhere on the landscape at each time step $(m=1 \cdot 0)$, but $g=0 \cdot 2$. Lighter shades of grey represent the highest resource levels, while increasingly depleted resources are represented by darker shades of grey and eventually black. The model scenarios shown are for 144 consumers at $t=2000$. (e) Covariance in resource abundance $\left(\times 10^{10}\right)$ with distance from a focal cell for the foraging types shown in (a)-(d). 


\section{1}

Resource

heterogeneity and individual variability

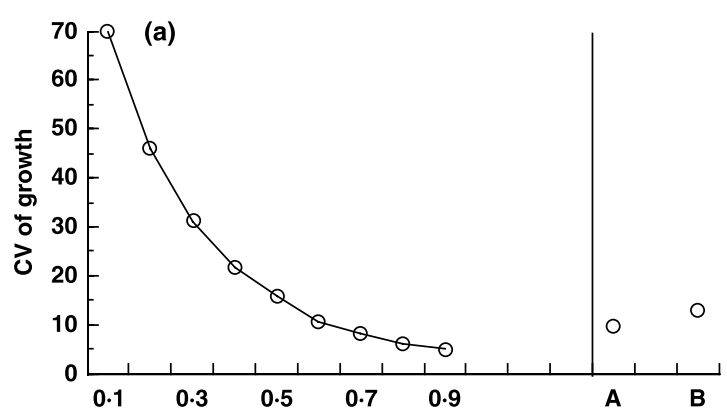

(b)

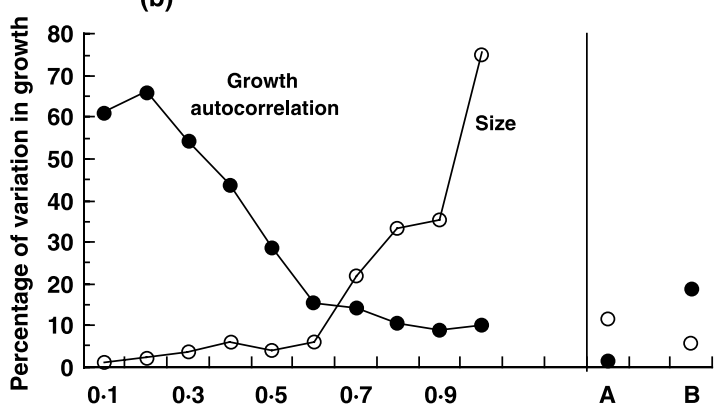

(c)

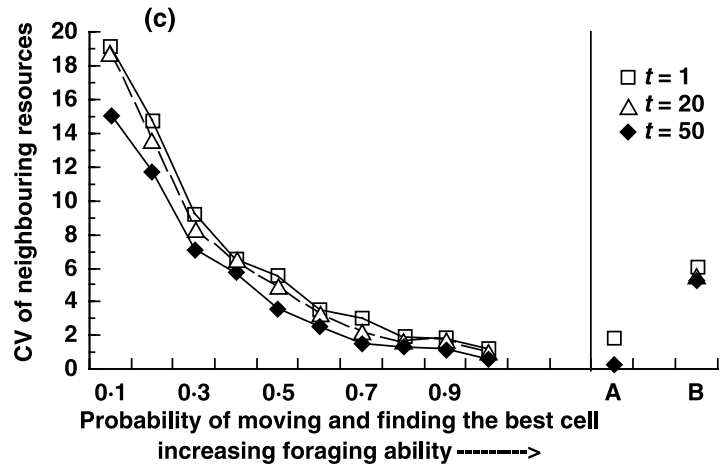

Fig. 4. (a) The CV of consumer growth as individuals increase in foraging ability ( $m$ and $g$ simultaneously and equally increased) in the model. A represents a 'highly mobile' forager where $m=1.0$ and $g=0.2$ and consumers move every time step to anywhere on the grid, while B differs only from A in that consumers can move only to neighbouring cells. In (b), the percentage of the variation in growth explained by size (open circles) and growth autocorrelation (filled circles), while (c) shows the variability among individuals (as CV) in the mean resource levels immediately surrounding a subset of 80 consumers. The data are summarized for one point in time $(t=2000)$, and an average over 10 and 50 time-steps.

('highly mobile' forager), a scenario associated with relatively homogeneous resources, resulted in low variability in growth and growth autocorrelation explaining only $1.9 \%$ of the growth (Fig. $4 \mathrm{a}, \mathrm{b}$, scenario A). When these same foragers were constrained to move to neighbouring cells only, variability among individuals in growth and the importance of growth autocorrelation increased an order of magnitude (Fig. 4a,b, scenario B).

The relationship between landscape patchiness and

(C) 2003 British

Ecological Society, Journal of Animal Ecology, 72, $725-735$ growth autocorrelation can be demonstrated by examining the resource levels each consumer encounters. For each of a random subset of 80 consumers on the grid, the mean resource level in the eight cells surround- ing each consumer showed greater variability among consumers as foraging ability declined (Fig. 4c). As consumers became increasingly better foragers and the landscape was less patchy, individuals encountered similar levels of resources in their immediate area. The patterns persisted when the mean neighbouring resource levels were averaged over 10 and 50 time steps (Fig. 4c), suggesting that poorer foragers are remaining in similar resource neighbourhoods throughout these longer time periods. Even when consumers moved every time step, but could only move to neighbouring cells, different individuals persistently encountered disparate resource environments (Fig. 4c, scenario B). As expected, based on the homogeneity of resources, the 'highly mobile' foragers encountered very similar resource levels (Fig. 4c, scenario A).

Growth patterns in a heterogeneous landscape could be influenced by both local resource levels and consumer foraging patterns. Hypothetically, frequent movers might encounter cells that are less depleted than stationary consumers and this might affect the estimation of autocorrelation. We examined this possibility using the 'poor forager' scenario $(m=0 \cdot 2$, $g=0 \cdot 2$ ), by quantifying how an individual's history of movement affected growth. Note that all consumers had identical probabilities of moving in our model, but some individuals will have moved in consecutive intervals while others will not have moved. A comparison showed that the growth of consumers in low resource environments (defined as the mean resource level in 8 surrounding cells) that did move (mean $=0.00141$ ) grew less than those consumers that did not move in a relatively high resource environment (mean = 0.00195), indicating the importance of resource level in influencing growth rates. Further, if movement patterns, independent of their effects on resource levels, were important to growth autocorrelation, then we would expect that solely the movement history of individuals could generate growth autocorrelation. Although movement added variation to the growth autocorrelation estimate, individuals that did not move for consecutive intervals could also be consistently better growers, while individuals that did move could be consistently poor growers. Thus, the spatial variation of resources that resulted from consumer foraging and movement, but not the movement differences directly, determined differences in growth and the strength of growth autocorrelation.

The model revealed that consumer foraging patterns interacted with density to affect the spatial heterogeneity of the resource and the importance of growth autocorrelation in individual consumers. When individuals were poor foragers, resource values among neighbouring cells covaried strongly over a range of densities (Fig. 5a), indicating persistent resource patchiness. In contrast, when individuals were good foragers, the covariance among cells was relatively small across all densities and distances (Fig. 5c), indicating a relatively homogeneous landscape. Intermediate foragers caused 
732

C. A. Pfister \&

S. D. Peacor
(C) 2003 British

Ecological Society, Journal of Animal Ecology, 72,

$725-735$ (a) Poor forager

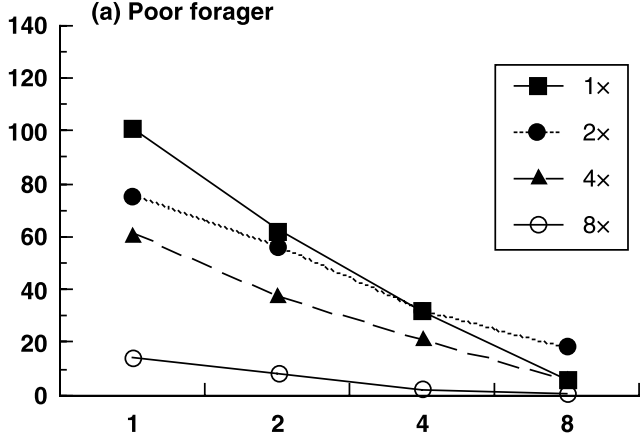

(b) Intermediate forager

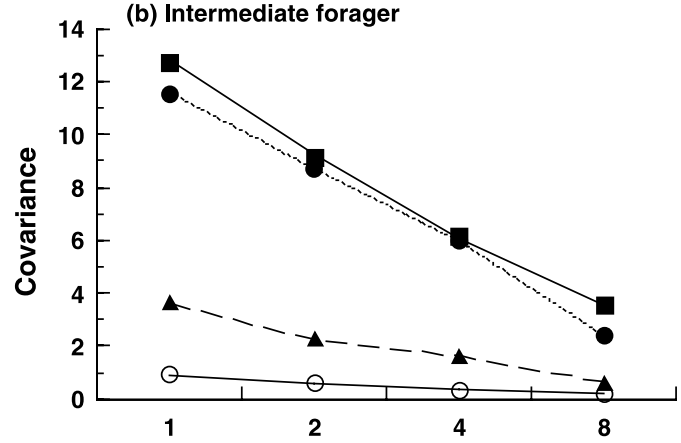

(c) Good forager

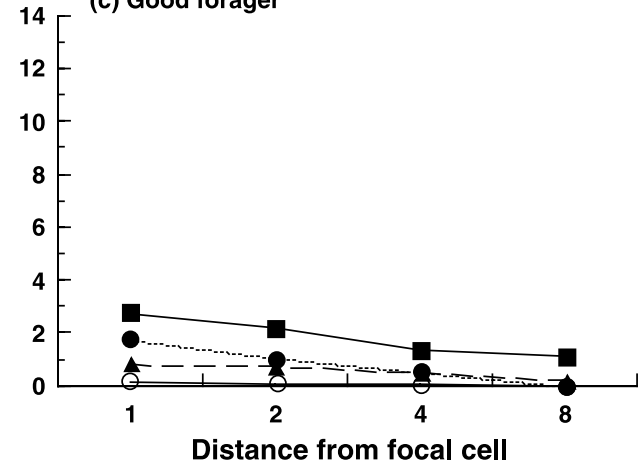

Fig. 5. Covariance in resource abundance $\left(\times 10^{10}\right)$ with distance from a focal cell on the model landscape for (a) poor, (b) intermediate and (c) good foragers, where density ranged eightfold. Note scale differences.

relatively high covariance among cells at low densities only, with covariance estimates declining with density (Fig. 5b). Thus, landscape patchiness was pronounced with poor foragers, but not good foragers, over a range of densities and with intermediate foragers at low densities only.

The effects of density on growth autocorrelation mirrored the effects on resource patchiness. Previous growth contributed relatively little to the variation in growth of good foragers across all densities, whereas it always caused more than half the variation in the growth of poor foragers, regardless of density. In contrast, density had a large effect on the contribution of autocorrelation to total growth variation for intermediate foragers. Increasing the density of consumers with intermediate foraging ability both decreased resource patchiness (Fig. 5) and weakened the role of previous growth (Fig. 6). Thus, consumers that moved and found the highest resource cells with intermediate

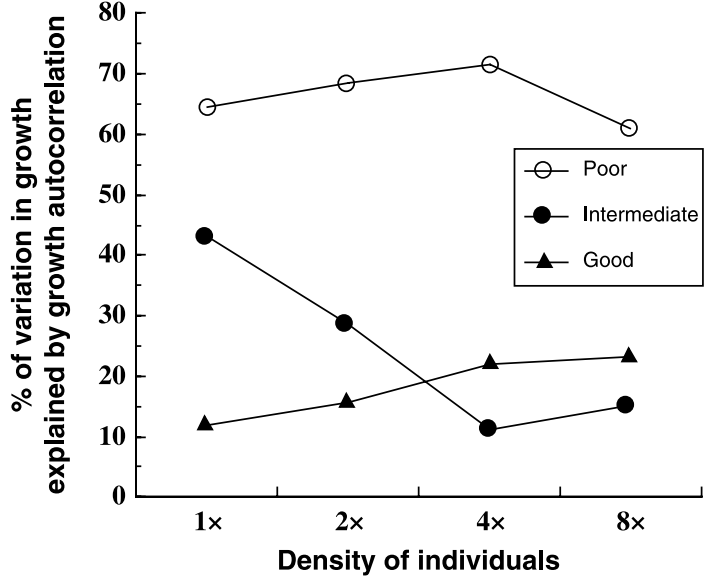

Fig. 6. The percentage of variation in growth explained by growth autocorrelation as population density increases in the model for individuals with intermediate foraging abilities. The foraging types are described in Fig. 3.

probabilities produced resource patchiness and exhibited high autocorrelation in growth through time. At higher densities, however, patchiness declined and growth autocorrelation was weakened.

\section{Discussion}

Our model results show that growth autocorrelationcan develop in populations without invoking size-dependent advantages, intrinsic trait differences among individuals, or extrinsic sources of environmental heterogeneity. Rather, the interaction between the individual foragers and the environment can cause spatial heterogeneity in resources to develop, causing positive correlations in individual growth. The model also highlights the importance of consumer foraging patterns, including their mobility and the extent to which consumers can identify rich resource cells. As long as consumers can move frequently across the landscape, regardless of their ability to find rich resource cells, resources will be relatively homogeneous (compare Fig. $3 \mathrm{c}$ with $3 \mathrm{~d}$ ). In the case of this 'highly mobile' consumer, variability in growth is described primarily by the size of the consumer; thus, size-dependent processes are sufficient to explain the growth of consumers. As the ability of consumers to both find rich resource cells and move in the landscape declines, resource patchiness develops with some areas more heavily foraged to low resource levels while others remain resource rich. Even in the case of consumers that move constantly, the inability to find the best resource cells coupled with movement that is restricted to neighbouring cells results in some heterogeneity in resources encountered (Fig. 4c, scenario B) and the contribution of growth autocorrelation to explaining $20 \%$ of the variation in growth (Fig. 4b, scenario B).

Consumers that are poorer foragers then encounter resource patchiness differentially (Fig. 4c), such that some consumers may remain in relatively resource rich 


\section{Resource}

heterogeneity and individual

variability
(C) 2003 British

Ecological Society,

Journal of Animal

Ecology, 72,

$725-735$ patches while others continue foraging in relatively resource poor areas. Thus, the local movement of imperfect foragers leads to individuals moving within disparate resource patches and increases the importance of growth autocorrelation.

In our model, we varied the foraging movement patterns of individuals by varying their ability to move and to find high resource cells, which in turn affected resource heterogeneity. We found that the resource heterogeneity was highest for the 'poorer' foragers. Because these foragers were also most likely to be in relatively poor patches, their distribution clearly departs the most from optimal foraging models (e.g. Charnov 1976), such as the ideal free distribution (IFD; Fretwell 1972). There are several mechanisms proposed by which animals are likely to deviate from an IFD, including risks of predation, disturbance and inability to locate high resources (Abrahams \& Dill 1989; Kennedy et al. 1994). While in the model we manipulated the ability to locate resources, adding predation risk or disturbance would increase deviations from the IFD in much the same way as a reduced ability to find high resources or limited mobility. Therefore we expect that other mechanisms that increase deviations from the IFD will also increase correlations in individual growth over time, as estimated by growth autocorrelation. Indeed, estimates of growth autocorrelation could reveal consistencies and deviations from the predictions of optimal foraging theories such as the IFD.

The presence of autocorrelation in T. scutum in tidepools suggests that limpets may remain in resourcepoor patches at the expense of poor growth. If lower movement rates underlie the origin of growth autocorrelation, then it may be due to either a lack of knowledge about resource levels elsewhere or to behavioural constraints to movement. In the terminology of the IFD, our data suggest that this limpet population is unlikely to be either 'ideal' or 'free' based on the presence of growth autocorrelation. Note that in the model, only 'good' foragers ( $m$ and $g$ are high) are behaving in a way that approaches optimality and might approximate an IFD. When foragers are 'good', rich resource patches in the landscape are immediately exploited, variability in growth among consumers is low and growth autocorrelation is trivial.

The effect of population density in our field experiment was to decrease the strength of growth autocorrelation; we had an analogous result in the model when consumers were 'intermediate' in their foraging abilities. One explanation for this is that growth is simply so suppressed at higher densities that there is little variation and growth autocorrelation cannot be manifested. However, this explanation is at odds with our data and growth patterns hinted at in previous empirical work. First, our limpets did grow at higher densities, but at only half the rate of those at reduced densities. Secondly, growth variability, estimated as a CV, was identical for limpets in the two treatments. Finally, there was still variation in individual growth as population density increased in our model. What differed is the extent to which different factors explained variability in growth. As density increased, growth autocorrelation explained less variation in growth for both intermediate and good consumers, but size explained increasingly more of the variation in growth. These patterns may be analogous to those seen in plants where dominance and suppression is observed at high densities (Harper 1977; Cousens \& Hutchings 1983; Weiner 1985; Schmitt et al. 1986, 1987; Dean et al. 1989; Reed 1990). However, the mechanism underlying patterns of individual variation in plants may differ from those modelled in our study. A recent model of plant dynamics suggests that asymmetric competitive interactions may generate dominant and suppressed individuals (Weiner et al. 2001). In contrast, there were no such asymmetries among individual consumers in our model; only the size of consumers and their interaction with their resource generated individual growth differences. Although the mobile consumers we modelled are not analogous to sessile plants, the entire gradient of mobility in animal consumers may be informative in understanding growth variability. In more sessile organisms, growth autocorrelation may increase in strength due the inability of the organism to homogenize resource use in space and time. Here the mechanism underlying the patterns of growth autocorrelation in the experimental manipulation with limpets is unknown to us; however, our model results suggest that the interaction between limpets and their resource may provide an explanation for increasing growth autocorrelation with decreasing density.

Although our experimental data with limpets does not provide a test of our consumer-resource model, the model does capture the foraging attributes of T. scutum and their potential for affecting growth autocorrelation. T. scutum is a generalist herbivore that grazes on multiple types of algae in a two-dimensional environment (e.g. crustose and filamentous algae) (Kitting 1980). Additionally, resources and consumers in the model shared many characteristics with our experimental system: resources had a refuge and were renewable, and consumers were not territorial and shared the same foraging area (Kitting 1980). Although our model findings highlight the importance of quantifying both resource heterogeneity and the precise pattern of consumer foraging to understand the origin of growth autocorrelation, we do not have this information for tidepool limpets. For many systems, including intertidal pools, where the spatial and temporal dynamics of resources will be difficult to quantify, the model mechanism will remain suggestive. An alternative explanation exists for the patterns of limpet growth that we demonstrated in the experiment. Individual limpets may have had intrinsic differences that affected foraging or conspecific interactions; differences that were expressed only at low density and were absent at control densities. We cannot rule out the existence of this mechanism. 
734

C. A. Pfister \&

S. D. Peacor
Our model results, in tandem with other published results quantifying growth autocorrelation (Pfister \& Stevens 2002), suggest that the mobility of individuals in a population is an important determinant to the patterns of variation in growth and size. Species with reduced mobility, either behaviourally or physiologically, may have strong growth autocorrelation, either due to extrinsic environmental heterogeneities or because their interaction with a uniform resource can readily generate patchiness. A number of consumerresource models show a rich variety of spatial structure (Hassell, Comins \& May 1994; Maron \& Harrison 1997; Wilson 1998; Abrams 2000; Wilson \& Richards 2000). A common theme in many of these models is that limited movement or dispersal can generate strong spatial structure. Our model complements these by showing that spatial resource pattern can greatly affect the attributes of and variation among individuals on a rapid time-scale (within a generation). With a greater emphasis on the collection and analysis of data at the level of the individual, we can further understand the causes and population consequences of differences among individuals.

\section{Acknowledgements}

This project was funded by grants from NSF (OCE9711802) and the Mellon Foundation to C. A. P. We thank R. Kordas, A. Miller, K. Rose, J. Salamunovitch, B. Scott, F. Stevens and L. Weis for help in the field and R. Riolo for help with the model. J. T. Wootton, P. Amaresekare, G. Dwyer, M. Leibold and two anonymous reviewers made helpful comments on the manuscript. C. A. P. thanks the Makah Tribal Nation for access to their lands.

\section{References}

Abrahams, M.V. \& Dill, L.M. (1989) A determination of the energetic equivalence of the risk of predation. Ecology, 70, 999-1007.

Abrams, P.A. (2000) The impact of habitat selection on the spatial heterogeneity of resources in varying environments. Ecology, 81, 2902-2913.

Arnold, S.J. (1981) Behavioral variation in natural populations: 2. The inheritance of a feeding response in crosses between geographic races of the garter snake, Thamnophis elegans. Evolution, 35, 510-515.

Caswell, H. (2001) Matrix Projection Models. Sinauer Press, Sunderland, MA.

Charnov, E.L. (1976) Optimal foraging: the marginal value theorem. Theoretical Population Biology, 9, 129136.

Connor, M.M. \& White, G.C. (1999) Effects of individual heterogeneity in estimating the persistence of small populations. Natural Resource Modeling, 12, 109-127.

Coulsen, T., Catchpole, E.A., Albon, S.D., Morgan, B.J.T., Pemberton, J.M., Clutton-Brock, T.H., Crawley, M.J. \& Grenfell, B.T. (2001) Age, sex, density, winter weather, and population crashes in Soay Sheep. Science, 292, 1528-1531.

Cousens, R. \& Hutchings, M.J. (1983) The relationship between density and mean frond weight in monospecific seaweed stands. Nature, 301, 240-241.
Darwin, C. (1859) The Origin of Species. John Murray, London.

Dean, T.A., Thies, K. \& Lagos, S.L. (1989) Survival of juvenile giant kelp: the effects of demographic factors, competitors, and grazers. Ecology, 70, 483-495.

DeAngelis, D.L., Rose, K.A., Crowder, L.B., Marschall, E.A \& Lika, D. (1993) Fish cohort dynamics: application of complementary modeling approaches. American Naturalist, 142, 604-622.

Deevey, E.S. (1947) Life tables of natural populations of animals. Quarterly Review of Biology, 22, 283-314.

Dukas, R. \& Bernays, E.A. (2000) Learning improves growth rate in grasshoppers. Proceedings of the National Academy of Sciences USA, 97, 2637-2640.

Fox, G.A. \& Kendall, B.E. (2002) Demographic stochasticity and the variance reduction effect. Ecology, 83, 1928-1934.

Fraser, D.F., Gilliam, J.F., Daley, M.J., Le, A.N. \& Skalski, G.T. (2001) Explaining leptokurtic movement distributions: intrapopulation variation in boldness and exploration. American Naturalist, 158, 124-135.

Fretwell, S.D. (1972) Populations in a Seasonal Environment. Princeton University Press, New Jersey.

Garćia-Berthou, E. (2001) On the misuse of residuals in ecology: testing regression residuals vs. the analysis of covariance. Journal of Animal Ecology, 70, 708-711.

Gill, S.K. \& Schultz, J.R., eds. (2000) Tidal Datums and Their Application. NOAA Special Publication, NOS CO-OPS 1. US Department of Commerce, Silver Spring, MD.

Harper, J.L. (1977) The Population Biology of Plants. Academic Press, London.

Hassell, M.P., Comins, H.N. \& May, R.M. (1994) Species coexistence and self-organizing spatial dynamics. Nature, 370, 290-292.

Hatch, S.A. (1990) Individual variation in behavior and breeding success of Northern Fulmars. Auk, 107, 750-755.

Kendall, B.E. \& Fox, G.A. (2002) Variation among individuals and reduced demographic stochasticity. Conservation Bio$\log y$, 16, 109-116.

Kennedy, M., Shave, C.R., Spencer, H.G. \& Gray, R.D. (1994) Quantifying the effect of predation risk on foraging bullies: no need to assume an IFD. Ecology, 75, 2220-2226.

Kitting, C.L. (1980) Herbivore-plant interactions of individual limpets (Acmaea scutum) maintaining a mixed diet of intertidal marine algae. Ecological Monographs, 50, 527550.

Lomnicki, A. (1980) Regulation of population density due to individual differences and patchy environment. Oikos, 35, 185-193.

Lomnicki, A. (1988) Population Ecology of Individuals. Princeton University Press, Princeton, NJ.

Maron, J.L. \& Harrison, S. (1997) Spatial pattern formation in an insect host-parasitoid system. Science, 278, 16191621.

Palmer, A.R. (1983) Growth rate as a measure of food value in Thaidid gastropods: assumptions and implications for prey morphology and distribution. Journal of Experimental Marine Biology and Ecology, 73, 95-124.

Palmer, A.R. (1984) Prey selection by thaidid gastropods: some observational and experimental field tests of foraging models. Oecologia, 62, 162-172.

Peckarsky, B.L. \& Cowen, C.A. (1991) Consequences of larval intraspecific competition to stonefly growth and fecundity. Oecologia, 88, 277-288.

Pfister, C.A. \& Stevens, F.R. (2002) The genesis of size variability in plants and animals. Ecology, 83, 59-72.

Pfister, C.A. \& Stevens, F.R. (2003) Individual variation and environmental stochasticity: implications for matrix model predictions. Ecology, 84, 496-510.

Reed, D.C. (1990) An experimental evaluation of density dependence in a subtidal algal population. Ecology, 71, $2286-2296$. 


\section{5}

Resource

heterogeneity and

individual

variability
Rossi, R.E., Mulla, D.J., Journel, A.G. \& Franz, E.H. (1992) Geostatistical tools for modeling and interpreting ecological spatial dependence. Ecological. Monographs, 62, 277-314.

Rubenstein, D.I. (1981) Individual variation and competition in the Everglades pygmy sunfish. Journal of Animal Eco$\log y, \mathbf{5 0}, 337-350$.

Schmitt, J., Eccleston, J. \& Ehrhardt, D.W. (1987) Dominance and suppression, size-dependent growth and self-thinning in a natural Impatiens capensis population. Journal of Eco$\log y, \mathbf{7 5}, 651-665$.

Schmitt, J., Ehrhardt, D.W. \& Cheo, M. (1986) Light-dependent dominance and suppression in experimental radish populations. Ecoloogy, 67, 1502-1507.

Uchmanski, J. (2000) Individual variability and population regulation: an individual-based model. Oikos, 90, 539-548.

Weiner, J. (1985) Size hierarchies in experimental populations of annual plants. Ecology, 66, 743-752.

Weiner, J., Stoll, P., Muller-Landau, H. \& Jasentuliyana, A.
(2001) The effects of density, spatial pattern, and competitive symmetry on size variation in simulated plant populations. American Naturalist, 158, 438-450.

Werner, E.E. (1992) Individual behavior and higher-order interactions. American Naturalist, 140, S5-S32.

West, L. (1986) Interdividual variation in prey selection by the snail Nucella (= Thais) emarginata. Ecology, 67, 798-809.

Wilbur, H.M. \& Collins, J.P. (1973) Ecological aspects of amphibian metamorphosis. Science, 182, 1305-1314.

Wilson, W.G. (1998) Resolving descrepancies between deterministic population models and individual-based simulations. American Naturalist, 151, 116-134.

Wilson, W.G. \& Richards, S.A. (2000) Evolutionarily stable strategies for consuming a structured resource. American Naturalist, 155, 83-100.

Ziemba, R.E. \& Collins, J.P. (1999) Development of size structure in tiger salamanders: the role of intraspecific interference. Oecologia, 120, 524-529.

Received 13 September 2002; accepted 11 April 2003
(C) 2003 British

Ecological Society, Journal of Animal Ecology, 72,

$725-735$ 